\title{
Illuminating Disorder Induced by Glu in a Stable Arg-Anchored Transmembrane Helix
}

Jake R. Price, Fahmida Afrose, Denise V. Greathouse and Roger E. Koeppe II*

Department of Chemistry and Biochemistry, University of Arkansas, Fayetteville, AR 72701

\section{Supporting information}

Figure S1. HPLC purification of the synthesized peptide $\mathrm{R}^{14} \mathrm{E}^{16} \mathrm{GWALP} 23$.

Figure S2. MALDI-TOF mass spectrum for the synthesized peptide $\mathrm{R}^{14} \mathrm{E}^{16} \mathrm{GWALP23}$.

Figure S3. $\mathrm{pH}$ dependence for $\mathrm{R}^{14} \mathrm{E}^{16} \mathrm{GWALP} 23$ in DOPC. 


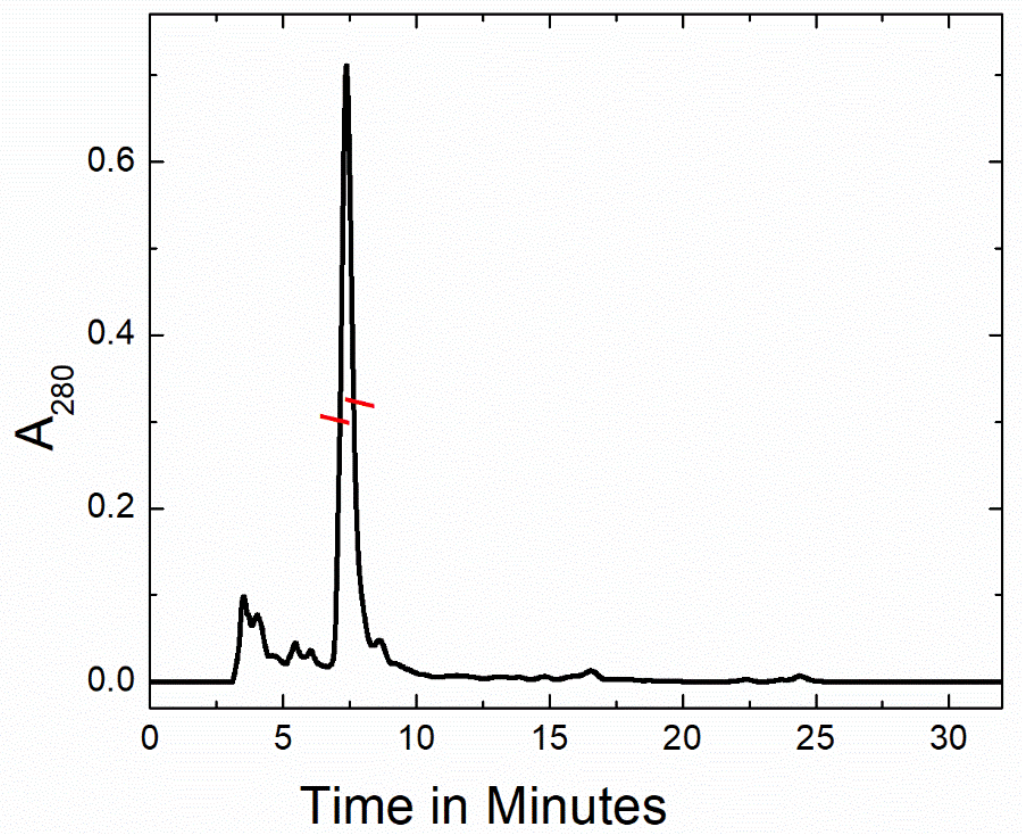

Figure S1. Reversed-phase HPLC purification of $R^{14} E^{16} G W A L P 23$ with deuterated alanines $A 11$ and $A 13$. An octyl-silica column (Zorbax Rx-C8, $9.4 \times 250 \mathrm{~mm}, 5 \mu \mathrm{m}$ particle size) was eluted using a gradient of 88-92\% methanol over 30 min. The main peak was separated from incompletely synthesized shorter peptides and collected to a purity of $>95 \%$ as indicated by the red segments. 


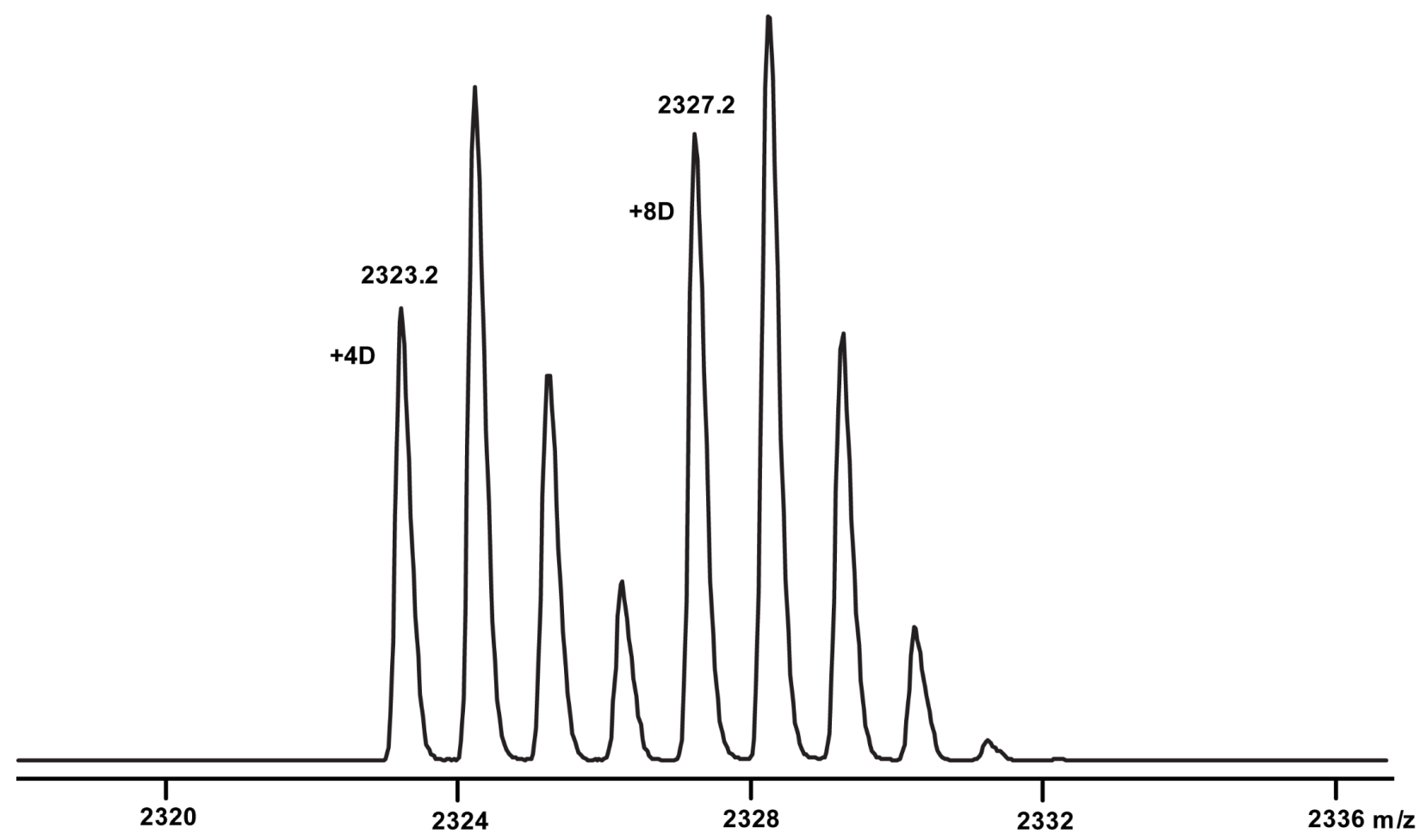

Figure S2. Isotope distribution from MALDI-TOF mass spectrum for the synthesized $R^{14} E^{16} G W A L P 23$ peptide. The spectrum confirms the molecular weight and deuterium distribution of the peptide with two alanines deuterated at levels of $50 \%$ and $100 \%$. The expected monoisotopic $\mathrm{m} / \mathrm{z}$ value for the $\left[\mathrm{M}+\mathrm{H}^{+}\right]$ion without ${ }^{13} \mathrm{C}$ is 2319.3 . The observed values with 4 or 8 deuterons agree with predictions. Success peaks in each envelope differ by $+/$ - one ${ }^{13} \mathrm{C}$ atom. 


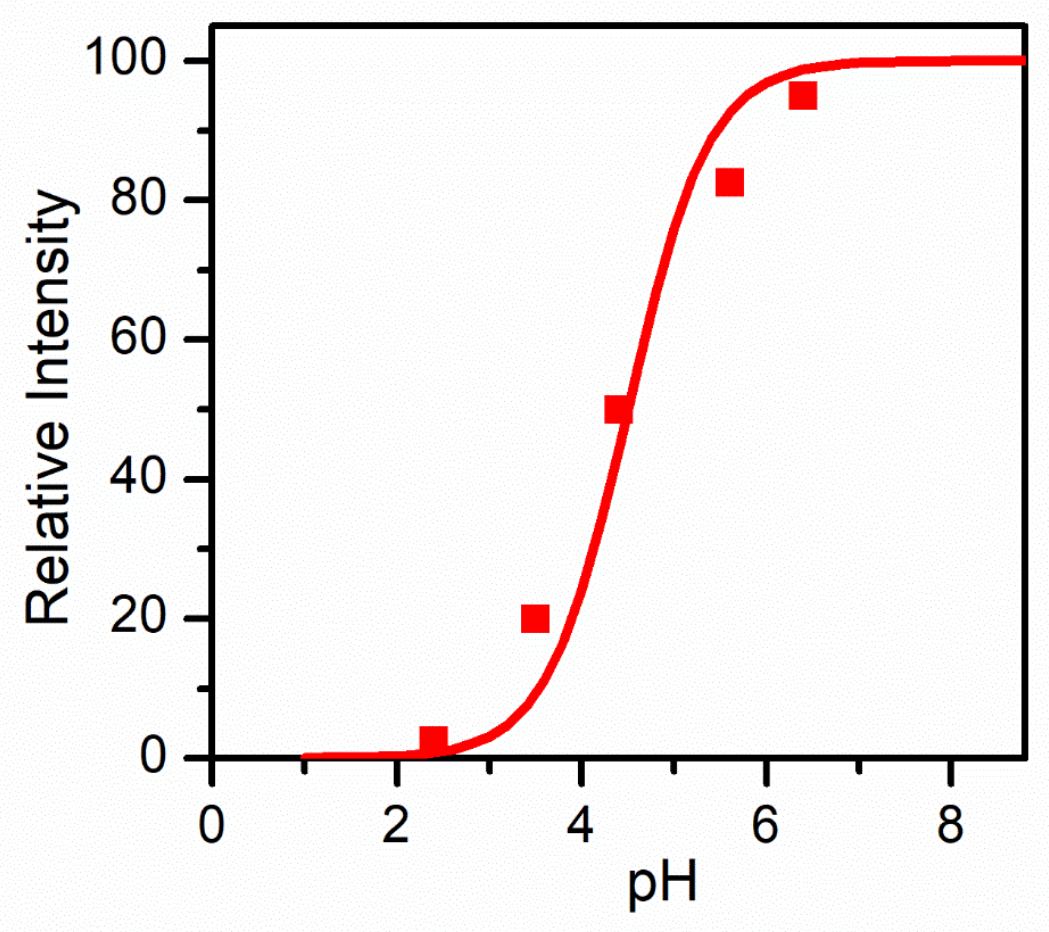

Figure S3. $\mathrm{pH}$ dependence for the appearance of a resonance at about $20 \mathrm{kHz}$ in the ${ }^{2} \mathrm{H}$ NMR spectra for oriented samples of $\mathrm{R}^{14} \mathrm{E}^{16} \mathrm{GWALP} 23$ in DOPC, when residues 11 and 13 are labeled with deuterium. 